\title{
Simulation and experimental investigation of a parallel serpentine-baffle flow field plate against fuel crossover in a direct ethanol proton exchange membrane fuel cell
}

\author{
G. Benetti ${ }^{1}$, E. Mathias ${ }^{1}$, P. M. Belchor ${ }^{1}$ and M.M.C. Forte $^{2}$ \\ ${ }^{1} \mathrm{ACET}, \mathrm{UNOESC}$ \\ Campus II of Joaçaba - Rua José Firmo Bernardi, Joaçaba (Brazil) \\ Phone/Fax number:+0055 49 35512000, e-mail: pablo.belchor@gmail.com \\ ${ }^{2} \mathrm{PPGE} 3 \mathrm{M}, \mathrm{UFRGS}$ \\ Campus Vale - Agronomia, Porto Alegre (Brazil) \\ Phone/Fax number:+0055 5133089475
}

\begin{abstract}
The negative effects of ethanol crossover through the electrolyte membrane are potential reduction and cathode depolarization, which decrease the overall efficiency of the direct ethanol proton exchange membrane fuel cell (DEPEMFC). In this paper, different combinations of two flow field plates, parallel serpentine-baffle (PSBFFP) and parallel serpentine (PSFFP), in the anode and cathode is investigated aiming of minimizing ethanol crossover in the cell. The ethanol crossover is evaluated by simulating the fuel flow and experimental test in DE-PEMFC prototype unit. The results show that ethanol crossover through the membrane electrolyte assembly (MEA) is minimized when the anode and cathode were fitted with PSFFP and PSBFFP, respectively. The crossover reduction is due to the high oxygen gas pressure in the discontinuous channels of PSBFFP in contact with MEA on the cathode side. On the other hand, no ethanol molecule reaches the cathode by crossover at the continuous channel of PSBFFP, which is the fuel cell outlet and has low pressure. The water produced by the redox reaction exits the cell through the low-pressure continuous channel, which improves the overall fuel cell performance.
\end{abstract}

\section{Key words}

Fuel cells, flow field plate, ethanol, crossover, simulation.

\section{Introduction}

One of the main drawbacks that limit the application of DE-PEMFCs as a competitive device is the ethanol crossover from the anode to the cathode side of the cell [1]. The ethanol crossover causes depolarization loss at the cathode decreasing the potential, thereby decreasing cell efficiency during operation. Generally, the ethanol crossover rate is affected by temperature, current density, ethanol feed concentration [2], and the maximum rate occurs when the cell operates at open-circuit voltage. The ethanol crossover rate decreases as the cell current density increases because more ethanol molecules are electrooxidized at anode compartment, and due to the ethanol concentration difference in the anode and cathode.

The performance of the DE-PEMFC was evaluated according to the operating parameters effect on the crossover rate of ethanol, reaching good agreement between the theoretical and experimental results.

\section{Objectives}

The aim of this research was evaluated both PSBFFP and PSFFP flow field plates with regard to fuel crossover using computational fluid dynamic (CFD) simulation. CFD tests were carried out to detail the reagents' flow behaviour in both anode and cathode electrodes. Experimental data obtained in a DE-PEMFC prototype unit fitted with Nafion ${ }^{\circledR}$ membrane provided a baseline for validation of the fuel cell theoretical efficiency according to the flow field design.

\section{Methodology}

The CFD experiments were carried out independently for the anode and cathode to evaluate the reagent fluid dynamic behaviour. Fluid dynamic simulations were performed using the flow simulation tool of the SOLIDWORKS 2013 software installed in the Alienware Aurora Desktop - BRH3171 (3.2 GHz, 8 MB L3 cache; 24GB DDR3 1333MHz; memory 6x4GB) equipped with Intel $^{\circledR}$ Core $^{\mathrm{TM}}$ i7-960 and a high-performance liquid cooler (Alienware®). Two conditions were simulated for the anode and cathode corresponding to the use of the PSBFFP or PSFFP. In the anode side, water at room temperature $\left(25^{\circ} \mathrm{C}\right)$ was used instead of the $1 \mathrm{M}$ ethanol aqueous solution used in the experimental runs. Water was fed in the flow field inlet at a flow rate of 12 $\mathrm{mL} / \mathrm{min}$. In the cathode side, the fluid dynamic behaviour was simulated using oxygen at $25^{\circ} \mathrm{C}$ feed in the flow field inlet at a flow rate of $1 \mathrm{~L} / \mathrm{min}$. For all simulated runs the flow field plates temperature was $80^{\circ} \mathrm{C}$ and the outlet cell pressure was assumed to be room pressure. 
The experimental runs were carried out in a DE-PEMFC prototype unit, as shown in the schematic diagram in Figure 1. The prototype unit was fed with $1 \mathrm{M}$ ethanol aqueous solution in the anode and oxygen in the cathode. A peristaltic pump and a flow meter controlled the reagents at a fixed stoichiometry. The flow rates of ethanol and oxygen were $12 \mathrm{~mL} / \mathrm{min}$ and $1 \mathrm{~L} / \mathrm{min}$, respectively. The temperature of the DE-PEMFC unit was controlled by heaters connected to both flow field plates with a thermostat fixed in one of them, in thermal equilibrium with the other. The heaters and thermostat were connected to a temperature control system (Model: CTT-13). The dynamic load (Model: fpc13a-2b) was connected to the DE-PEMFC unit and to a computer. The overall temperature of the DE-PEMFC unit was measured by thermocouples located on opposite sides of each flow field plate. FUTURE-S\&T (SC/BR) company manufactured the dynamic load and temperature control system, and software specifically for this study. Although a DEPEMFC can produce a current density of $190 \mathrm{~mA} / \mathrm{cm}^{2}$, the maximum dynamic load current was purposely set to approximately $0.62 \mathrm{~A}$. The maximum current density measured in the tests, for current of $0.62 \mathrm{~A}$ and flow field area of $9.0 \mathrm{~cm}^{2}$, was $70 \mathrm{~mA} / \mathrm{cm}^{2}$, when the fuel cell performance is more susceptive to the fuel crossover. By varying the ohmic resistance of the dynamic load, the electric current $I$ could be checked along with the corresponding electric potential $V$, and the current-voltage and current-power curves could be obtained. Figure 1 shows the Schematic DE-PEMFC unit flow diagram.

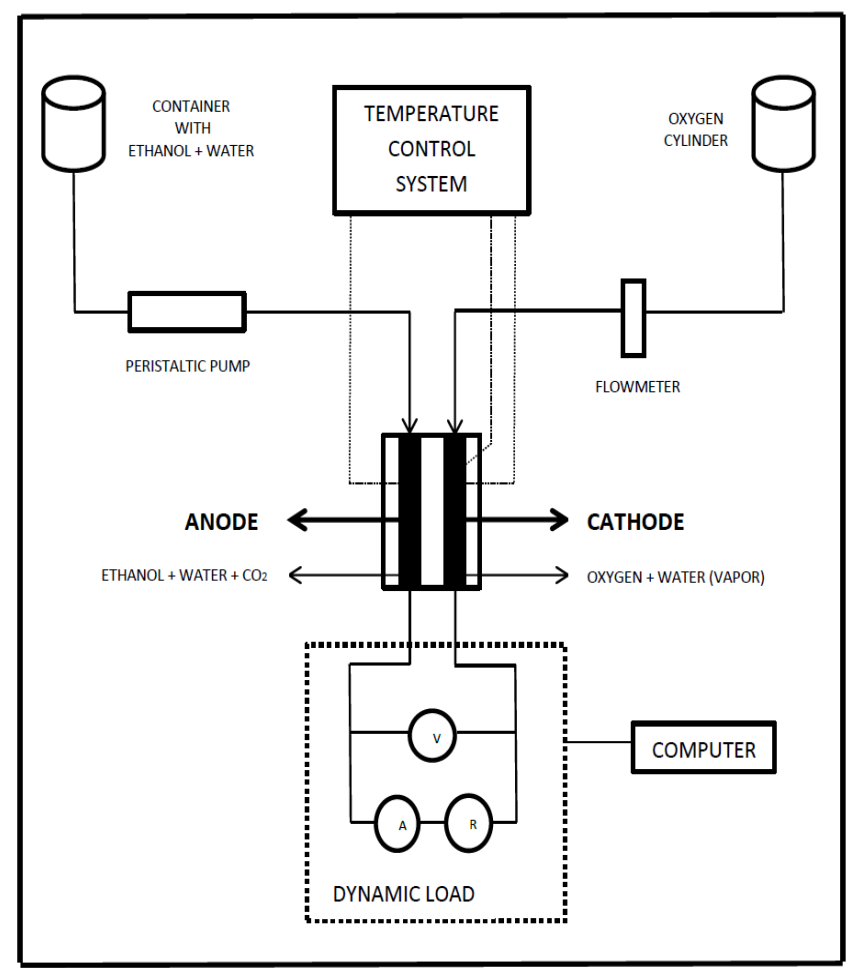

Fig. 1. Schematic DE-PEMFC unit flow diagram.

The runs in the DE-PEMFC unit with Nafion membrane were carried out using four different combinations of the plates PSFFP (A) and PSBFFP (B) in the anode and cathode sides as shown in Figure 2. To guarantee maximum crossover during the test, the ethanol concentration was $1 \mathrm{M}$ and the fuel cell temperature was $80^{\circ} \mathrm{C}$.

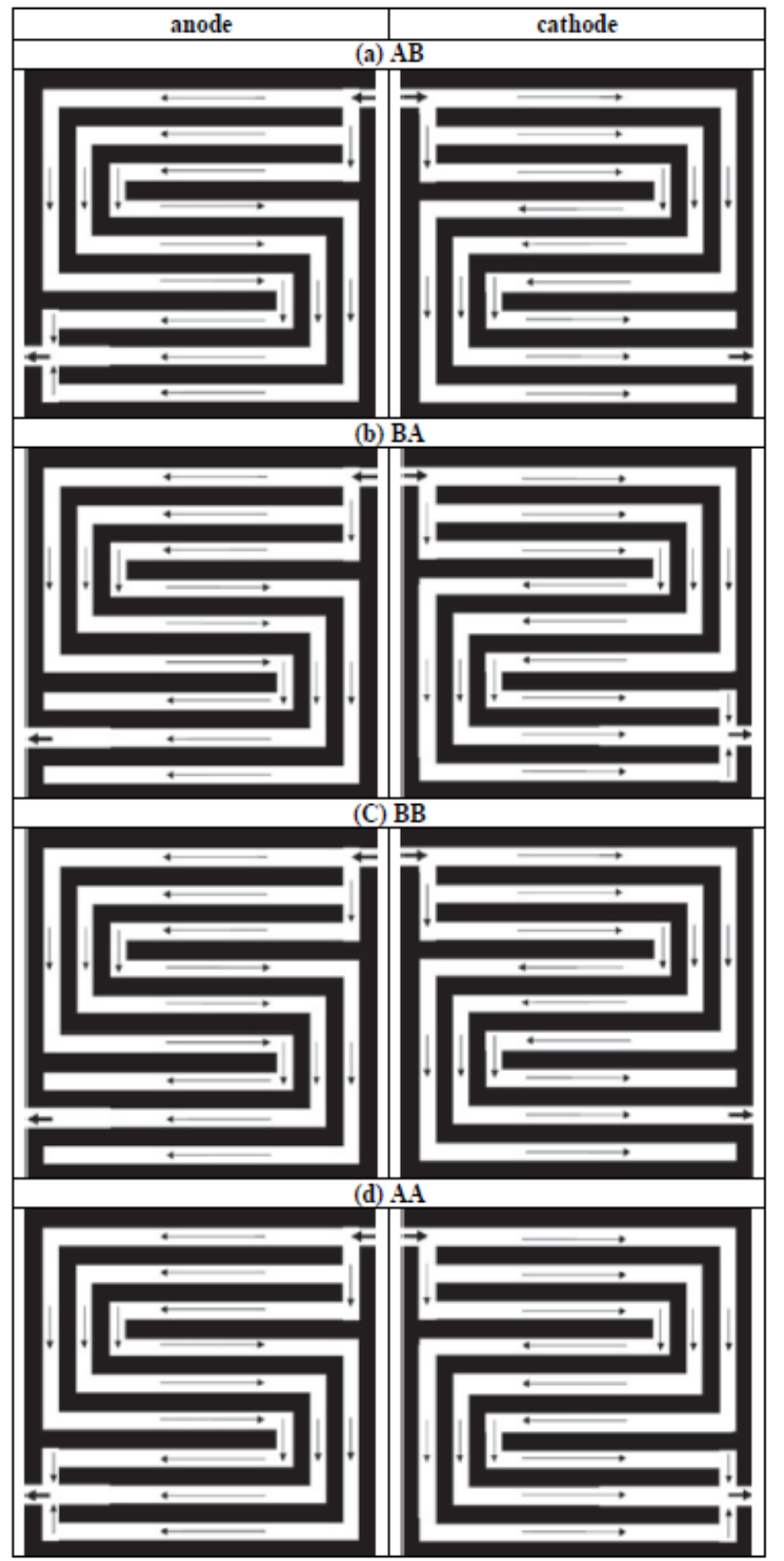

Fig. 2. Schematic diagram of the four combinations of flow field plates, (A) PSFFP and (B) PSBFFP, in the anode/cathode. (a) $\mathrm{A} / \mathrm{B}$; (b) $\mathrm{B} / \mathrm{A}$; (c) $\mathrm{B} / \mathrm{B}$; (d) $\mathrm{A} / \mathrm{A}$.

\section{Results and Discussion}

The flow field simulations of water behaviour in the anode side equipped with either PSFFP or PSBFFP are shown in Figures 3 and 4, respectively. The pressure loss from the inlet to the outlet decreased from 100,862 $\mathrm{Pa}$ to $101,241 \mathrm{~Pa}$ in PSFFP and from 100,889 $\mathrm{Pa}$ to $101,271 \mathrm{~Pa}$ in PSBFFP, although it was already reported that serpentine flow channels have large pressure drops due to long flow paths. The fluid velocity in PSFFP was at the maximum value $(0.136 \mathrm{~m} / \mathrm{s})$ near the inlet and outlet of the plate, and homogeneously distributed throughout the three channels. In both baffle channels of PSBFFP the fluid was static while in the serpentine channels the fluid behaviour was uniform between the inlet and outlet with maximum velocity of $0.115 \mathrm{~m} / \mathrm{s}$. 

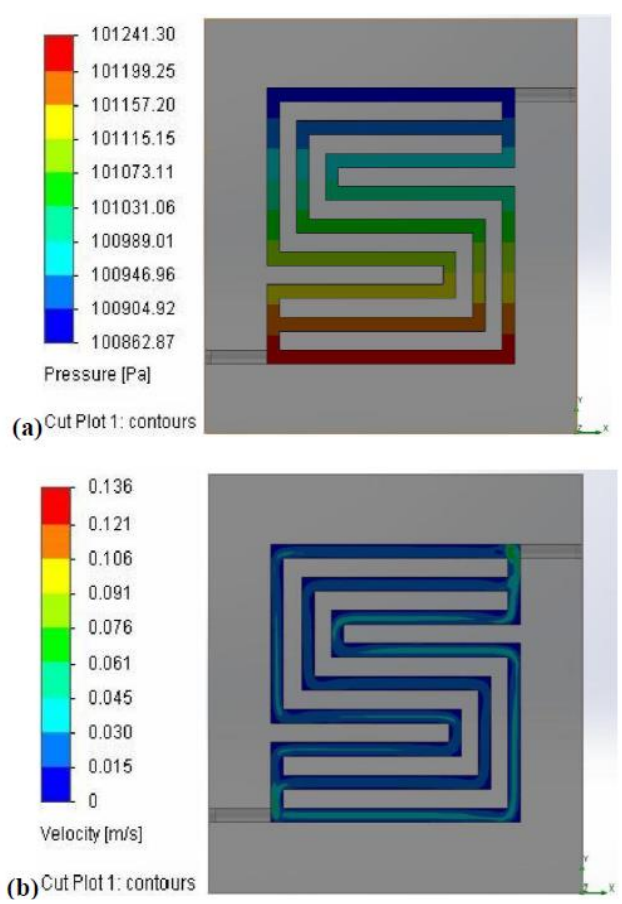

Fig. 3. Water flow simulation with PSFFP $\left(80^{\circ} \mathrm{C}\right)$ at the anode side of DE-PEMFC (water flow rate of $12 \mathrm{~mL} / \mathrm{min}$ at the inlet; environmental pressure at the outlet): (a) Pressure; (b) Velocity.

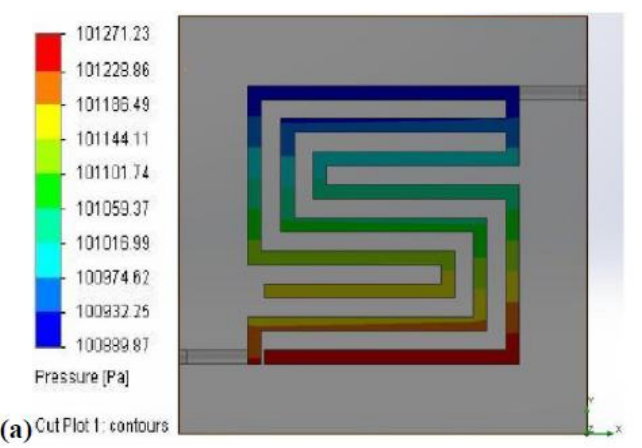

$$
\begin{aligned}
& 0.115 \\
& 0.102 \\
& 0.089 \\
& -0.077 \\
& 0.064 \\
& 0.051 \\
& -0.038 \\
& 0.026 \\
& 0.013 \\
& 0 \\
& \text { velocily }[\mathrm{mis} \mid
\end{aligned}
$$

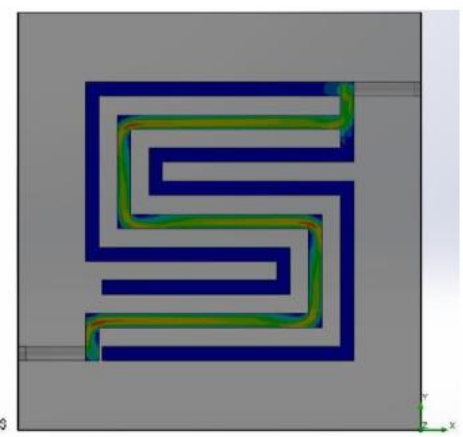

(b) CutPlot 1: contours

Fig. 4. Water flow simulation with $\operatorname{PSBFFP}\left(80^{\circ} \mathrm{C}\right)$ at the anode side of DE-PEMFC (water flow rate of $12 \mathrm{~mL} / \mathrm{min}$ at the inlet; environmental pressure at the outlet): (a) Pressure; (b) Velocity.

The flow simulations of oxygen behaviour in the cathode side equipped with either PSFFP or PSBFFP are shown in Figures 5 and 6, respectively. At the cathode side, the flow field design had an influence on oxygen pressure distribution. The pressure loss increased from the inlet $(101,578 \mathrm{~Pa})$ to the outlet $(101,312 \mathrm{~Pa})$ in the cathode fitted with PSFFP, as noted by other researchers. The fluid velocity was at a maximum value of $13,278 \mathrm{~m} / \mathrm{s}$ close to the inlet and outlet, and homogeneously distributed throughout the three channels.
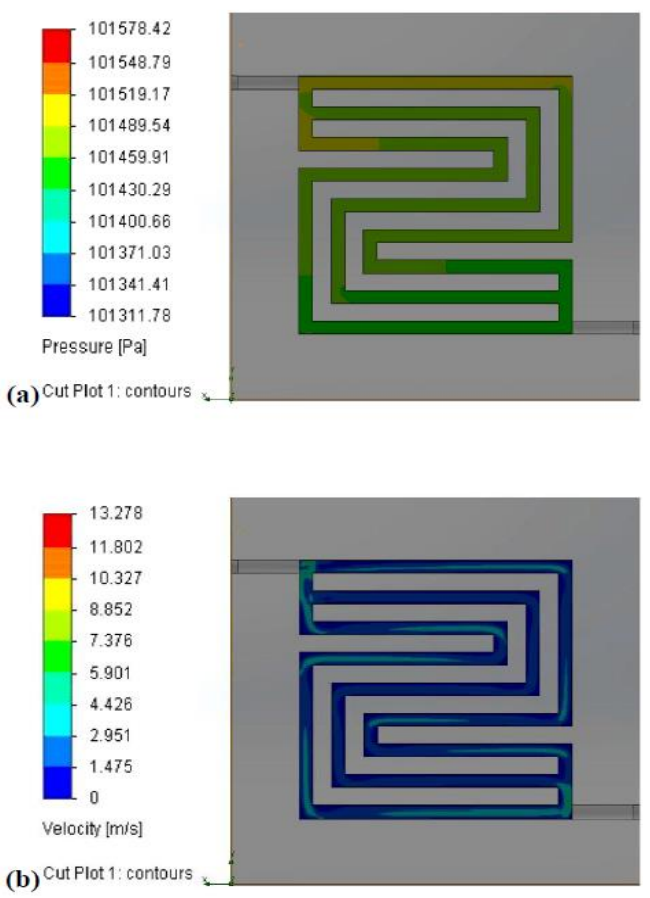

Fig. 5. Oxygen flow simulation with PSFFP $\left(80^{\circ} \mathrm{C}\right)$ at the cathode side of DE-PEMFC (oxygen flow rate of $1 \mathrm{~L} / \mathrm{min}$ at the inlet; environmental pressure at the outlet): (a) Pressure; (b) Velocity.

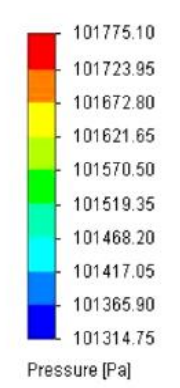

(a) Cut Plot 1: contours
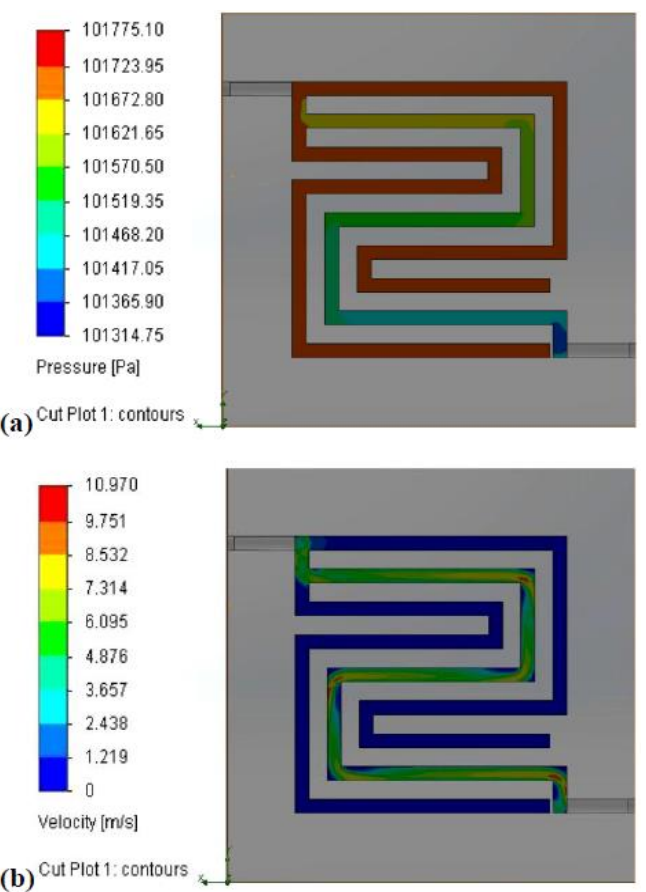

Fig. 6. Oxygen flow simulation with PSBFFP $\left(80^{\circ} \mathrm{C}\right)$ at the cathode side of DE-PEMFC (oxygen flow rate of $1 \mathrm{~L} / \mathrm{min}$ at the inlet; environmental pressure at the outlet): (a) Pressure; (b) Velocity.

The oxygen gas acquired complete velocity and pressure loss stability in the cathode with PSBFFP. In the baffle channels, the gas velocity was null and the pressure was maximum $(101,775 \mathrm{~Pa})$. On the other hand, in the serpentine channels, the gas pressure loss increased from the inlet $(101.775 \mathrm{~Pa})$ to the outlet $(101.315 \mathrm{~Pa})$, and the oxygen reached the maximum velocity of $10.970 \mathrm{~m} / \mathrm{s}$. In particular, the flow field geometry has an important effect on the mass transport rates of reactants and 
products and on the pressure drop of the reactants across the cell.

The experimental curves of voltage and power versus current for the four combinations of PSBFFP and PSFFP are shown in Figure 7. The DE-PEMFC unit performance in the current range of 0 to $0.6 \mathrm{~A}$ varied according to the plates' combination at the anode and cathode. Above 0.1 A, the cell fitted with PSFFP in the anode and PSBFFP in the cathode (red curve) presented the best performance among the four combinations. Using the plates combination PSFFP/PSBFFP, the cell reached a maximum power of $0.16 \mathrm{~W}$, which is twice the maximum power $(0.075 \mathrm{~W})$ reached by the cell equipped with the inverse combination (PSBFFP/PSFFP). For the cells equipped with PSFFP/PSFFP or PSBFFP/PSBFFP, the maximum power achieved was $0.07 \mathrm{~W}$ and $0.05 \mathrm{~W}$, respectively, and the current values were not higher than $0.4 \mathrm{~A}$.
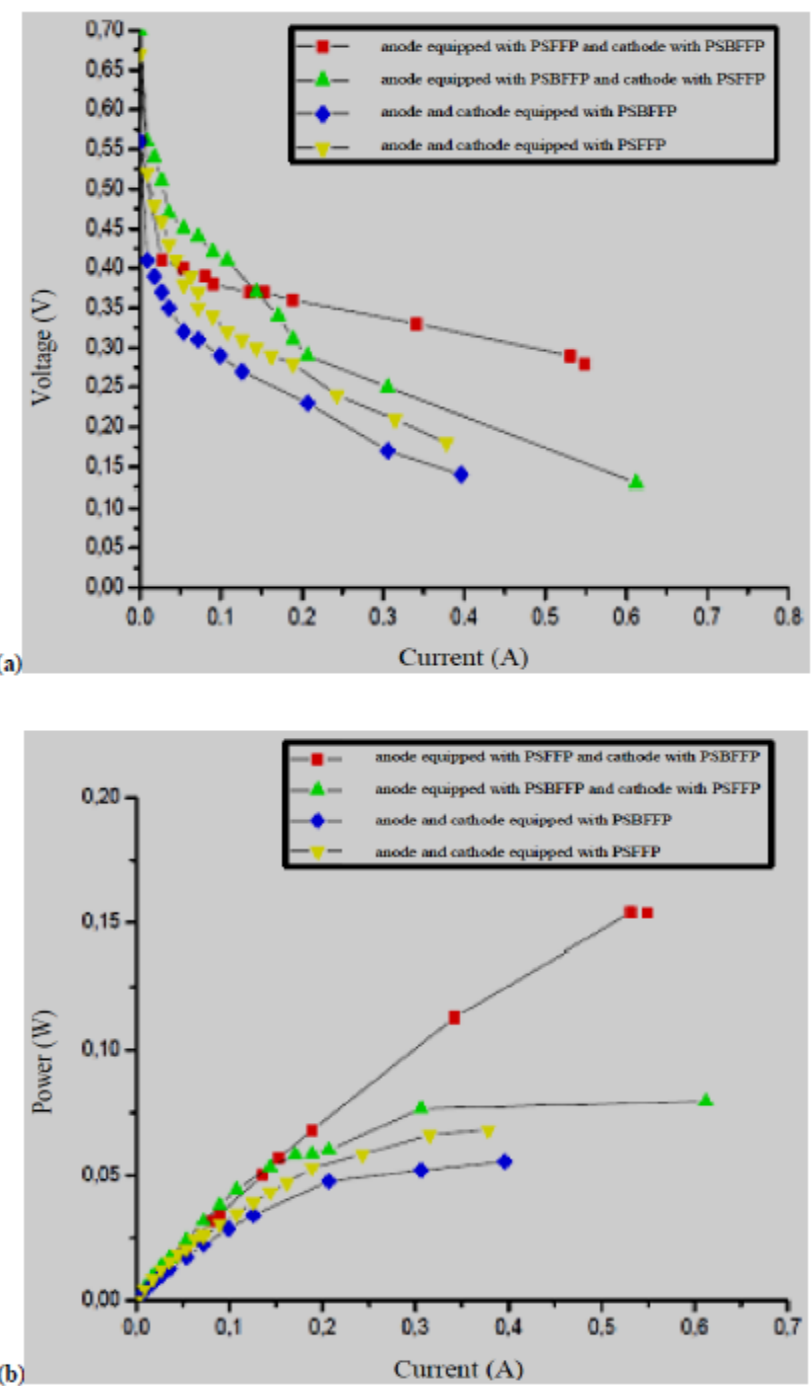

Fig. 7. Curves of (a) current-voltage and (b) current-power obtained in the DE-PEMFC prototype for the four combinations of flow field plates in the anode/cathode.

The fuel cell fitted with PSBFFP/PSFFP (green curve) has showed better performance at currents below $0.1 \mathrm{~A}$, where activation overpotential occur. The activation overpotential is directly related to reaction kinetics, and the electrochemical reactions propagate under a rate demanded by a specific load. As reported in literature [3], the oxygen reduction kinetic by a $\mathrm{Pt}$ electrocatalyst is excellent compared to that of ethanol electro-oxidation by a Ptbased electrocatalyst. Thus, it is assumed that the activation overpotential at the cathode is less significant compared to that at the anode. Beyond this low-current region associated with activation overpotential, there is a strong interference of ethanol crossover and the fuel cell fitted with PSFFP/PSBFFP showed the best performance. The maximum power density reached by the DE-PEMFC prototype fitted with the four different combinations of flow field plates in the anode and cathode is shown in Figure 8. The DE-PEMFC prototype fitted at the anode with PSFFP and at the cathode with PSBFFP reached the highest maximum power, which was $92 \%$ and $165 \%$ higher, respectively, of the maximum power obtained when the cell was fitted with PSBFFP/PSFFP and only with PSBFFP. The maximum power difference when the fuel cell was fitted only with PSFFP was $120 \%$. The results show that the use of the baffle plate PSBFFP is strategic if a better fuel cell performance is sought.

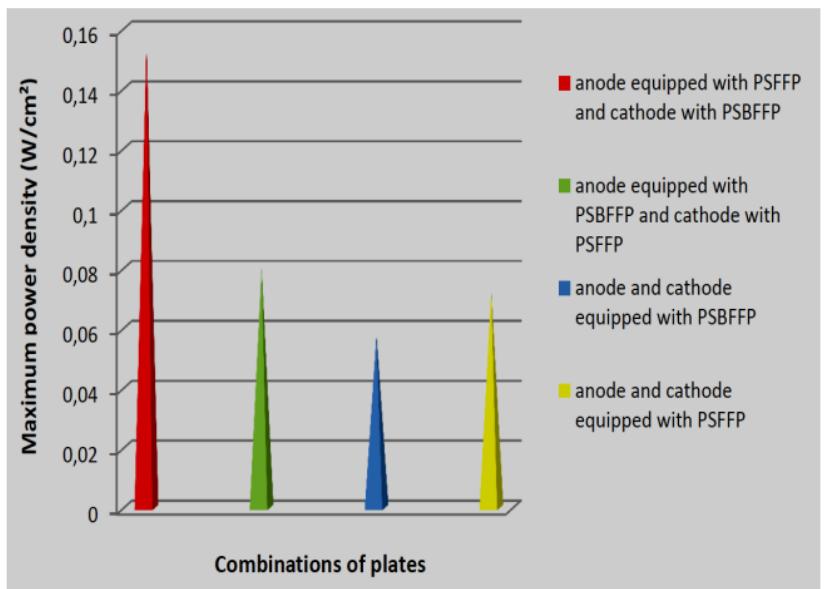

Fig. 8. Maximum power density vs. combination type of cell.

Nguyen [4] has showed in the study of a gas distributor design for PEMFC that an interdigitated flow field plate pushes the reagents more strongly through the diffusion layer. By the fluid dynamic simulation tests was seen that through the two interdigitated channels of PSBFFP, there was a much higher oxygen pressure on the MEA at the cathode side compared to the pressure exerted by the alcohol on the same MEA at the anode side. The results here discussed are in accordance with that presented by Nguyen since the PSBFFP has changed the fuel cell performance considerably depending on which plate is fitted in the fuel cell sides. Computations showed that the pressure loss relative to the unique serpentine channel on the PSBFFP at the cathode was at a maximum throughout the cell $(\Delta \mathrm{p} \approx-460 \mathrm{~Pa})$. However, the increase in pressure loss within this channel promoted convective or under-rib flow. This flow favoured elimination of the residual water in the cathode side and increased the overall efficiency of the electrochemical reaction, and a maximum current density was obtained. 


\section{Conclusion}

In the present research, a perfect balance between decreasing the ethanol crossover and efficient removal of the water produced at the cathode of a DE-PEMFC was achieved without the use of peripherals, by combining different flow field plates, a PSFFP at the anode and a PSBFFP at the cathode. For the best fuel cell performance obtained, the major behavioural difference between the anode equipped with PSFFP and the cathode equipped with PSBFFP was due to the pressure of the reagent within the channels of each plate. The reagents were pushed more intensely against the diffusion layer in the two discontinuous flow channels of the PSBFFP visualized through the fluid simulation tests. In the PSBFFP channels, the pressure exerted by oxygen on the MEA at the cathode is greater than that exerted by the alcohol on the same MEA at the anode. We conclude that a higher oxygen pressure at the cathode related to the alcohol pressure at the anode can decrease ethanol crossover in a DA-PEMFC. However, decreasing ethanol crossover is not a guarantee of improvement in the overall fuel cell performance; this must be combined with efficient removal of the water produced by redox reaction in the cathode.

\section{Acknowledgement}

The financial support of FUTURE - Science \& Technology is gratefully acknowledged.

\section{References}

[1] Y.S. Li, T.S. Zhao, Z.X. Liang, J Power Sources. 187 (2009) 387-392.

[2] G. Andreadis, S. Song, P. Tsiakaras, J Power Sources. 157 (2006) 657-665.

[3] Y. Takasu, T. Jwazaki, W. Sugimoto, Y. Marakami, Electrochem Commun.2 (2000) 671-674.

[4] T.V. Nguyen, J Electrochem Soc. 143 (1996) L103-L105. 